\title{
Limiting absorption principles for the Navier equation in elasticity
}

\author{
JuAn Antonio Barceló, Magali Folch-Gabayet, \\ Salvador PÉrez-Esteva, Alberto Ruiz and Mari Cruz Vilela
}

\begin{abstract}
We prove some a priori estimates for the resolvent of Navier equation in elasticity, when one approaches the spectrum (Limiting Absorption Principles). They are extensions of analogous estimates for the resolvent of the euclidean Laplacian in $\mathbb{R}^{n}$. As a consequence, we get some results for the evolution equation and for the spectral measure.
\end{abstract}

Mathematics Subject Classification (2010): 35J47 (primary); 74B05, 42B37 (secondary).

\section{Introduction and statement of results}

The Navier equation of the dynamic linearized elasticity in a homogeneous and isotropic medium is ruled by the wave operator

$$
\frac{\partial^{2}}{\partial t^{2}}-\Delta^{*}
$$

where the operator $\Delta^{*}$, acting on the $x$-variable of vector-valued functions $\mathbf{u}(x, t) \in$ $\mathbb{C}^{n}$ reads

$$
\Delta^{*} \mathbf{u}=\mu \Delta \mathbf{I} \mathbf{u}+(\lambda+\mu) \nabla \operatorname{div} \mathbf{u},
$$

and $\Delta \mathrm{I}$ denotes the diagonal matrix with the Laplace operator on the diagonal and $\lambda, \mu$ are the Lamé constants.

In the case of time harmonic solution of frequency $\omega$ the operator to consider, known as spectral Navier operator, is

$$
L \mathbf{u}(x)=\Delta^{*} \mathbf{u}(x)+\omega^{2} \mathbf{u}(x),
$$

where $\omega>0, x \in \mathbb{R}^{n}, n \geq 2$, and $\mathbf{u}$ is a vector-valued function from $\mathbb{R}^{n}$ to $\mathbb{C}^{n}$.

The first and fourth authors were supported by Spanish Grant MTM2008-02568, the second and the third by Proyecto CONACyT-DAIC U48633-F, and the fifth by Spanish Grant MTM200762186.

Received December 20, 2010; accepted April 19, 2011. 
Throughout this paper we will assume that $\mu>0$ and $2 \mu+\lambda>0$ so that the operator $\Delta^{*}$ is strongly elliptic and, we will denote by $k_{p}$ and $k_{s}$ respectively the speed of propagation of longitudinal and transversal waves, which are given by

$$
k_{p}^{2}=\frac{\omega^{2}}{(2 \mu+\lambda)} \quad \text { and } \quad k_{s}^{2}=\frac{\omega^{2}}{\mu} .
$$

It is well known that any solution $\mathbf{u}$, of the homogeneous spectral Navier equation

$$
L \mathbf{u}(x)=\Delta^{*} \mathbf{u}(x)+\omega^{2} \mathbf{u}(x)=\mathbf{0},
$$

with $\Delta^{*}$ given by (1.2), in a domain, can be written as the sum of the so called compressional part, denoted by $\mathbf{u}_{p}$, and the shear part, denoted by $\mathbf{u}_{s}$, where

$$
\mathbf{u}_{p}=-\frac{1}{k_{p}^{2}} \nabla \operatorname{div} \mathbf{u} \quad \text { and } \quad \mathbf{u}_{s}=\mathbf{u}-\mathbf{u}_{p} .
$$

Observe that $\mathbf{u}_{p}$ and $\mathbf{u}_{s}$ are solutions of the vectorial homogeneous Helmholtz equations $\Delta \mathbf{I}_{\mathbf{p}}(x)+k_{p}^{2} \mathbf{u}_{\mathbf{p}}(x)=\mathbf{0}$ and $\Delta \mathbf{I u}_{\mathbf{s}}(x)+k_{s}^{2} \mathbf{u}_{\mathbf{s}}(x)=\mathbf{0}$, respectively.

Besides, if $\mathbf{u}$ is an entire solution (i.e. a solution in the whole $\mathbb{R}^{n}$ ) of (1.4) satisfying the Kupradze outgoing radiation conditions:

$$
\begin{aligned}
\left(\partial_{r}-i k_{p}\right) \mathbf{u}_{p} & =\mathbf{o}\left(r^{-(n-1) / 2}\right), & r & =|x| \rightarrow \infty, \\
\left(\partial_{r}-i k_{s}\right) \mathbf{u}_{s} & =\mathbf{o}\left(r^{-(n-1) / 2}\right), & r & =|x| \rightarrow \infty,
\end{aligned}
$$

then, $\mathbf{u}=\mathbf{0}$ (see [19] for the three-dimensional case).

As a consequence, for a vector-valued function $\mathbf{f} \in \mathcal{C}_{0}^{\infty}$, if there exists a solution of the Navier equation

$$
\Delta^{*} \mathbf{u}(x)+\omega^{2} \mathbf{u}(x)=\mathbf{f}(x), \quad \omega>0, x \in \mathbb{R}^{n}, n \geq 2,
$$

satisfying the Kupradze outgoing radiation conditions (1.6) and (1.7), where $\mathbf{u}_{p}$ and $\mathbf{u}_{s}$ are given by (1.5) out of the support of $\mathbf{f}$, then the solution is unique.

Similar statements hold true for the Kupradze incoming radiation conditions

$$
\begin{array}{rlrl}
\left(\partial_{r}+i k_{p}\right) \mathbf{u}_{p} & =\mathbf{o}\left(r^{-(n-1) / 2}\right), & & r=|x| \rightarrow \infty \\
\left(\partial_{r}+i k_{s}\right) \mathbf{u}_{s}=\mathbf{o}\left(r^{-(n-1) / 2}\right), & r & =|x| \rightarrow \infty .
\end{array}
$$

Equation (1.8) and conditions (1.6) and (1.7) are genuine vectorial versions of the scalar Helmholtz equation, given by

$$
\Delta v(x)+k^{2} v(x)=g(x), \quad k>0, x \in \mathbb{R}^{n}, n \geq 2,
$$

and the Sommerfeld outgoing radiation condition

$$
\left(\partial_{r}-i k\right) v=o\left(r^{-(n-1) / 2}\right), \quad r=|x| \rightarrow \infty .
$$


Throughout this paper, given a Banach space of scalar valued functions

$$
B\left(\mathbb{R}^{n}\right)=\left\{g: \mathbb{R}^{n} \longrightarrow \mathbb{C}:\|g\|_{B}<\infty\right\},
$$

its vector-valued version will be denoted by

$$
\mathbf{B}\left(\mathbb{R}^{n}\right)=\left\{\mathbf{f}: \mathbb{R}^{n} \longrightarrow \mathbb{C}^{n}:\|\mathbf{f}\|_{\mathbf{B}}<\infty\right\},
$$

where $\|\mathbf{f}\|_{\mathbf{B}}=\||\mathbf{f}|\|_{B}$, and $|\mathbf{f}|^{2}=\left|\left(f_{1}, \ldots, f_{n}\right)\right|^{2}=\left|f_{1}\right|^{2}+\ldots+\left|f_{n}\right|^{2}$ and its dual space by $\mathbf{B}^{*}\left(\mathbb{R}^{n}\right)$.

Limiting absortion principles. If $z=\gamma+i \varepsilon$ belongs to the resolvent set of $\Delta^{*}$, i.e. $\varepsilon \neq 0$ (see (2.7) below), there exists a constant $c(z)>0$ independent of $\mathbf{f}$ such that the following estimate holds:

$$
\left\|\left(\Delta^{*}+z \mathrm{I}\right)^{-1} \mathbf{f}\right\|_{\mathbf{L}^{2}} \leq c(z)\|\mathbf{f}\|_{\mathbf{L}^{2}}
$$

where I denotes the identity matrix of order $n$. The constant $c(z)$ blows up as $z$ approaches the spectrum of $\Delta^{*}$, that is $\mathbb{R}^{+}$. An interesting question is the existence of Banach spaces $\mathbf{B}\left(\mathbb{R}^{n}\right)$ such that the estimate

$$
\left\|\left(\Delta^{*}+z \mathrm{I}\right)^{-1} \mathbf{f}\right\|_{\mathbf{B}^{*}} \leq c(z)\|\mathbf{f}\|_{\mathbf{B}}
$$

holds for $\mathbf{f} \in \mathbf{L}^{2}\left(\mathbb{R}^{n}\right) \cap \mathbf{B}\left(\mathbb{R}^{n}\right)$ with $c(z)>0$ a constant independent of $\mathbf{f}$ so that it does not blow up when $z$ approaches the spectrum of $\Delta^{*}$.

Furthermore, given any interval $[a, b] \subset(0, \infty)$ we look for the existence of a constant $c$, such that

$$
\sup _{\gamma \in[a, b]} \sup _{\varepsilon \in(0,1)}\left\|\left(\Delta^{*}+(\gamma \pm i \varepsilon) \mathrm{I}\right)^{-1}\right\|_{\mathbf{B}} \longrightarrow \mathbf{B}^{*}<c .
$$

Under these conditions, if $\mathbf{L}^{2}\left(\mathbb{R}^{n}\right) \cap \mathbf{B}\left(\mathbb{R}^{n}\right)$ is dense in $\mathbf{B}\left(\mathbb{R}^{n}\right)$, we may consider $\mathbf{R}(z)$ the extension to $\mathbf{B}\left(\mathbb{R}^{n}\right)$ of the operator $\left(\Delta^{*}+z \mathrm{I}\right)^{-1}$ defined in $\mathbf{L}^{2}\left(\mathbb{R}^{n}\right) \cap \mathbf{B}\left(\mathbb{R}^{n}\right)$. Following standard techniques (see [1, Theorem 4.1], and see also [20]), from (1.13) one should be able to prove that for $\mathbf{f} \in \mathbf{B}\left(\mathbb{R}^{n}\right)$,

$$
\mathbf{R}\left(\omega^{2}+i 0\right) \mathbf{f}:=\underset{z \rightarrow \omega^{2}, \Im z>0}{\operatorname{sim}} \mathbf{R}(z) \mathbf{f}
$$

exists in $\mathbf{B}^{*}\left(\mathbb{R}^{n}\right)$ and is a weak solution of equation (1.8), which satisfies

$$
\left\|\mathbf{R}\left(\omega^{2}+i 0\right) \mathbf{f}\right\|_{\mathbf{B}^{*}} \leq c\left(\omega^{2}\right)\|\mathbf{f}\|_{\mathbf{B}}
$$

We say that the weak limiting absorption principle holds for the operator $\Delta^{*}$ in the space $\mathbf{B}\left(\mathbb{R}^{n}\right)$ for Kupradze radiation conditions if (1.14) and (1.15) are satisfied and, furthermore, under the extra assumption $\mathbf{f} \in \mathbf{C}_{0}^{\infty}\left(\mathbb{R}^{n}\right) \cap \mathbf{B}\left(\mathbb{R}^{n}\right)$ one has that out of the support of $\mathbf{f}, \mathbf{R}\left(\omega^{2}+i 0\right) \mathbf{f}$ is the sum, as in (1.5), of $\mathbf{u}_{s}$ and $\mathbf{u}_{p}$, satisfying (1.6) 
and (1.7). We assume also the similar statement for the incoming conditions (1.9) and (1.10) when one takes

$$
\mathbf{R}\left(\omega^{2}-i 0\right) \mathbf{f}:=\underset{z \rightarrow \omega^{2}, \Im z<0}{\operatorname{sim}} \mathbf{R}(z) \mathbf{f} .
$$

These principles have interesting consequences for the behavior of solution of wave equation and they are the first step to be accomplished, if one wants to treat some inverse scattering problems for the elasticity equations (see for instance [16] and [28]).

Function Spaces. Let us start by introducing the spaces $\mathbf{B}\left(\mathbb{R}^{n}\right)$ in which we study the limiting absorption principle.

We will use the scalar valued space introduced in [17], that we will denote by $X^{*}\left(\mathbb{R}^{n}\right)$ and which is given by the following norm:

$$
\|v\|_{X^{*}}^{2}=\sup _{R>0} \frac{1}{R} \int_{B(0, R)}|v(x)|^{2} d x
$$

where $v$ is a function defined on $\mathbb{R}^{n}$ with values in $\mathbb{C}$. This space is a homogeneous version of the space considered in [1] and [2]. In fact, if we consider $v_{\lambda}(x)=$ $v(\lambda x)$, with $\lambda>0$, we have that

$$
\left\|v_{\lambda}\right\|_{X^{*}}^{2}=\lambda^{1-n}\|v\|_{X^{*}}^{2}
$$

We will replace the norm in its predual space by the equivalent expression:

$$
\|g\|_{X}=\sum_{j \in \mathbb{Z}}\left(2^{j+1} \int_{C_{j}}|g(x)|^{2} d x\right)^{1 / 2}
$$

where $g$ is a function defined on $\mathbb{R}^{n}$ with values in $\mathbb{C}$, and

$$
C_{j}=\left\{x \in \mathbb{R}^{n}: 2^{j}<|x| \leq 2^{j+1}\right\}, \quad j \in \mathbb{Z} .
$$

Given a nonnegative function $V$, we will also use the weighted $-L^{2}$ space $L^{2}(V)$ defined as the Lebesgue space $L^{2}\left(\mathbb{R}^{n}\right)$ with respect to the measure $V(x) d x$. We consider weights $V$ in several spaces: Morrey-Campanato classes, certain homogeneous Herz spaces and the space of functions with everywhere bounded X-ray transform.

For $\alpha>0$ and $1 \leq r \leq n / \alpha$, the Morrey-Campanato classes (see [6] and [29]) are given by

$$
\mathcal{L}^{\alpha, r}\left(\mathbb{R}^{n}\right)=\left\{V \in L_{\ell \text { oc }}^{r}\left(\mathbb{R}^{n}\right):\|V\|_{\mathcal{L}^{\alpha, r}}<\infty\right\}
$$

where

$$
\|V\|_{\mathcal{L}^{\alpha, r}}=\sup _{x \in \mathbb{R}^{n}, \rho>0} \rho^{\alpha}\left(\rho^{-n} \int_{B(x, \rho)}|V(y)|^{r} d y\right)^{1 / r} .
$$


Notice that some Lebesque spaces are included in these classes: $\mathcal{L}^{\alpha, n / \alpha}\left(\mathbb{R}^{n}\right)=$ $L^{n / \alpha}\left(\mathbb{R}^{n}\right)$ We also remark that for $r<n / \alpha$ the class $\mathcal{L}^{\alpha, r}\left(\mathbb{R}^{n}\right)$ contains the Lorentz space $L^{n / \alpha, \infty}\left(\mathbb{R}^{n}\right)$.

For $1 \leq p \leq \infty$, we define the spaces

$$
D_{p}\left(\mathbb{R}^{n}\right)=\left\{V \in L_{\ell \mathrm{oc}}^{p}\left(\mathbb{R}^{n} \backslash\{0\}\right):\|V\|_{D_{p}}<\infty\right\},
$$

where

$$
\begin{aligned}
\|V\|_{D_{p}} & =\sum_{j \in \mathbb{Z}} 2^{j(p-n) / p}\left\|V \chi_{j}\right\|_{L^{p}}, \quad \text { if } 1 \leq p<\infty, \text { and } \\
\|V\|_{D_{\infty}} & =\sum_{j \in \mathbb{Z}} 2^{j}\left\|V \chi_{j}\right\|_{L^{\infty}},
\end{aligned}
$$

with $\chi_{j}=\chi_{C_{j}}$, where $\chi_{E}$ denotes the characteristic function of the set $E$ and $C_{j}$ is given by (1.17).

From the definition, it is easy to prove that if $p_{1} \geq p_{2}$, then

$$
D_{p_{1}}\left(\mathbb{R}^{n}\right) \subseteq D_{p_{2}}\left(\mathbb{R}^{n}\right)
$$

Besides, if $V$ is a radial function, then $\|V\|_{D_{p}\left(\mathbb{R}^{n}\right)}=\|V\|_{D_{p}(\mathbb{R})}$ where, abusing notation, $V=V(x)=V(r)$, with $r=|x|$. In such a case, we will simply write $\|V\|_{D_{p}}$ to denote both, $\|V\|_{D_{p}\left(\mathbb{R}^{n}\right)}$ and $\|V\|_{D_{p}(\mathbb{R})}$.

We want to note that the spaces $X\left(\mathbb{R}^{n}\right)$ and $D_{p}\left(\mathbb{R}^{n}\right)$ are homogeneous Herz spaces, in fact, following the notation used in [13] and [21], we have that $X\left(\mathbb{R}^{n}\right)=$ $\dot{K}_{2}^{1 / 2,1}\left(\mathbb{R}^{n}\right), D_{p}\left(\mathbb{R}^{n}\right)=\dot{K}_{p}^{(p-n) / p, 1}\left(\mathbb{R}^{n}\right)$ if $1 \leq p<\infty$, and $D_{\infty}\left(\mathbb{R}^{n}\right)=\dot{K}_{\infty}^{1,1}\left(\mathbb{R}^{n}\right)$. that

We denote by $\mathcal{T}\left(\mathbb{R}^{n}\right)$ (see [4]) the class of nonnegative radial functions $V$ such

$$
\||| V \mid\|:=\sup _{\mu>0} \int_{\mu}^{\infty} \frac{r V(r)}{\left(r^{2}-\mu^{2}\right)^{1 / 2}} d r<\infty,
$$

where as before, abusing notation, $V=V(x)=V(r)$, with $r=|x|$. This is to say that the $X$-ray transform of the function $V$ is bounded everywhere.

In general, the limit

$$
\lim _{\delta \rightarrow 0+} \frac{1}{\delta} \int_{C_{\delta, L}} g(y) d y,
$$

defines the $X$-ray transform of a function $g \in L_{\ell \text { oc }}^{1}\left(\mathbb{R}^{n}\right)$ on the set of all lines $L$ in $\mathbb{R}^{n}$. Here $C_{\delta, L}=\left\{y \in \mathbb{R}^{n}: d(y, L)<\delta\right.$ and $\left.|x-y|<\delta^{-1}\right\}, L=\{x+t \omega / t \in \mathbb{R}\}$, $x \in \mathbb{R}^{n}, \omega \in S^{n-1}$ and $d(y, L)$ denotes the Euclidean distance between $y$ and $L$.

Note that if $V$ is a radial function, then there exists a positive constant $C$ independent of $V$ such that

$$
\|V\|\|\leq C\| V \|_{D_{p}}
$$

if and only if $p>2$ (see [4, Remark 1] and Remark 4.6 below). 
We will use the vector-valued version of some of the spaces introduced above, which will be denoted by $\mathbf{X}\left(\mathbb{R}^{n}\right), \mathbf{X}^{*}\left(\mathbb{R}^{n}\right)$ and $\mathbf{L}^{2}(V)$.

The following theorems extend known limiting absorption principles for the Helmholtz equation to the Navier equation. The theorems are extensions in the sense that the Helmholtz equation can be viewed as the particular case of the Navier equation when we take $\mu+\lambda=0$.

Theorem 1.1. Let $z=\gamma+i \varepsilon$ with $\varepsilon \neq 0, \frac{1}{p}+\frac{1}{q}=1$ with $\frac{2}{n+1} \leq \frac{1}{p}-\frac{1}{q} \leq \frac{2}{n}$ if $n>2$, or $\frac{2}{3} \leq \frac{1}{p}-\frac{1}{q}<1$ if $n=2$. Let $V_{1}$ be a nonnegative real valued function in $\mathcal{L}^{2, r}\left(\mathbb{R}^{n}\right)$ with $(n-1) / 2<r \leq n / 2$ and $n>2$, and let $V_{2}$ be a nonnegative radial function in $D_{\tilde{r}}\left(\mathbb{R}^{n}\right)$ with $2<\overline{\tilde{r}} \leq \infty$. If $\mathbf{f} \in \mathbf{L}^{2}\left(\mathbb{R}^{n}\right)$ then, there exists a constant $c>0$ independent of $z, \mathbf{f}, V_{1}$ and $V_{2}$ such that the following a priori estimates hold:

$$
\begin{aligned}
\left\|\left(\Delta^{*}+z \mathrm{I}\right)^{-1} \mathbf{f}\right\|_{\mathbf{X}^{*}} & \leq c|z|^{-1 / 2}\|\mathbf{f}\|_{\mathbf{X}}, \\
\left\|\left(\Delta^{*}+z \mathrm{I}\right)^{-1} \mathbf{f}\right\|_{\mathbf{L}^{q}} & \leq c|z|^{\frac{n}{2}\left(\frac{1}{p}-\frac{1}{q}\right)-1}\|\mathbf{f}\|_{\mathbf{L}^{p}}, \\
\left\|\left(\Delta^{*}+z \mathrm{I}\right)^{-1} \mathbf{f}\right\|_{\mathbf{L}^{2}\left(V_{1}\right)} & \leq c\left\|V_{1}\right\|_{\mathcal{L}^{2, r}\|\mathbf{f}\|_{\mathbf{L}^{2}\left(V_{1}^{-1}\right)},} \\
\left\|\left(\Delta^{*}+z \mathrm{I}\right)^{-1} \mathbf{f}\right\|_{\mathbf{L}^{2}\left(V_{2}\right)} & \leq c|z|^{-1 / 2}\left\|V_{2}\right\|_{D_{\tilde{r}}}\|\mathbf{f}\|_{\mathbf{L}^{2}\left(V_{2}^{-1}\right)}
\end{aligned}
$$

Besides, the weak limiting absorption principle for $\Delta^{*}$ holds in the spaces $\mathbf{X}\left(\mathbb{R}^{n}\right)$, $\mathbf{L}^{q}\left(\mathbb{R}^{n}\right), \mathbf{L}^{2}\left(V_{1}\right)$ and $\mathbf{L}^{2}\left(V_{2}\right)$ for Kupradze radiation conditions.

Theorem 1.2. Let $p$ such that $\frac{1}{n+1} \leq \frac{1}{p}-\frac{1}{2} \leq \frac{1}{n}$, and let $V_{1}$ be a nonnegative real valued function in $\mathcal{L}^{2, r}\left(\mathbb{R}^{n}\right)$ with $(n-1) / 2<r \leq n / 2$ and $n>2$, and let $V_{2}$ be a nonnegative radial function in $D_{\tilde{r}}\left(\mathbb{R}^{n}\right)$ with $2<\tilde{r} \leq \infty$. If $\mathbf{f} \in \mathbf{C}_{0}^{\infty}\left(\mathbb{R}^{n}\right)$ and we consider the unique solution $\mathbf{u}=\left(u_{1}, \ldots, u_{n}\right)$ of equation (1.8) satisfying (1.6) and (1.7), then, there exists a constant $c>0$ independent of $\omega, \mathbf{f}, V_{1}$ and $V_{2}$ such that the following a priori estimates hold:

$$
\begin{aligned}
\sup _{1 \leq j \leq n}\left\|\nabla u_{j}\right\| \mathbf{X}^{*} & \leq c\|\mathbf{f}\|_{\mathbf{X}}, \\
\sup _{x_{0}, R>0} \frac{1}{R} \int_{B\left(x_{0}, R\right)}|\mathbf{u}(x)|^{2} d x & \leq c \omega^{2 n\left(\frac{1}{p}-\frac{1}{2}\right)-3}\|\mathbf{f}\|_{\mathbf{L}^{p}}^{2}, \\
\sup _{x_{0}, R>0} \frac{1}{R} \int_{B\left(x_{0}, R\right)}\left|D^{1 / 2} \mathbf{u}(x)\right|^{2} d x & \leq c\left\|V_{1}\right\|_{\mathcal{L}^{2, r}\|\mathbf{f}\|_{\mathbf{L}^{2}\left(V_{1}^{-1}\right)}^{2},} \\
\sup _{1 \leq j \leq n}\left\|\nabla u_{j}\right\|_{\mathbf{L}^{2}\left(V_{2}\right)} & \leq c\left\|V_{2}\right\|_{D_{\tilde{r}}}\|\mathbf{f}\|_{\mathbf{L}^{2}\left(V_{2}^{-1}\right)},
\end{aligned}
$$

where $D^{1 / 2} \mathbf{u}=\left(D^{1 / 2} u_{1}, \ldots, D^{1 / 2} u_{n}\right)$.

Remark 1.3. Estimates (1.23) and (1.27) are weaker than those known for the Helmholtz equation (see (3.10), (3.14) and (1.19)). We will explain later why in this case we could not extend the known results for the Helmholtz equation to the 
Navier equation (see Remark 3.11). A related open problem in harmonic analysis is the behavior of the space $\mathcal{T}\left(\mathbb{R}^{n}\right)$ with respect to the Hardy-Littlewood maximal operator.

Remark 1.4. The result given in (1.23) is sharp in the sense that the estimate is false for $\tilde{r} \leq 2$. We give the details in the last section.

Remark 1.5. It would be interesting to give an alternative proof of estimates (1.24) and (1.20) with the multiplier method in [22].

We recall certain well-known notions from the work of Kato [15] and Kato a Yajima [16]. Let $H$ be a self adjoint operator in a Hilbert space $\mathcal{H}$, so that the resolvent $\mathcal{R}_{H}(z)=(H-z)^{-1}$ is defined at least for $\Im z \neq 0$.

We say that a densely defined closed linear operator $T$ from $\mathcal{H}$ into itself is $H$-supersmooth if

$$
\left|\left\langle\mathcal{R}_{H}(z) T^{*} f, T^{*} f\right\rangle_{\mathcal{H}}\right| \leq c\|f\|_{\mathcal{H}}^{2} \quad f \in \mathbf{D}\left(T^{*}\right),
$$

uniformly in $\Im z \neq 0$. If $T$ satisfies the weaker condition

$$
\left|\mathfrak{s}\left\langle\mathcal{R}_{H}(z) T^{*} f, T^{*} f\right\rangle_{\mathcal{H}}\right| \leq c\|f\|_{\mathcal{H}}^{2} \quad f \in \mathbf{D}\left(T^{*}\right),
$$

uniformly in $\Im z \neq 0$, we say that $T$ is $H$-smooth. Here $T^{*}$ is the adjoint of $T$ and $\mathbf{D}\left(T^{*}\right)$ is the domain of $T^{*}$.

From the point of view of the Schrödinger evolution, (1.29) is equivalent to the estimate

$$
\int_{-\infty}^{\infty}\left\|T e^{i t H} f\right\|_{\mathcal{H}}^{2} d t \leq 2 \pi c\|f\|_{\mathcal{H}}^{2} \quad f \in H .
$$

If $\mathbf{R}(z)=\left(\Delta^{*}+z \mathbf{I}\right)^{-1}$ and $V$ is a nonnegative real function in $\mathcal{L}^{2, \alpha}(\mathbb{R})^{n},(n-1) / 2<$ $r \leq n / 2$, estimate (22) of Theorem 1.1 , which is equivalent to the uniform bound

$$
\left|\left\langle\mathbf{R}(z)\left(V^{1 / 2} \mathbf{f}\right), V^{1 / 2} \mathbf{f}\right\rangle_{\mathbf{L}^{2}}\right| \leq c\|\mathbf{f}\|_{\mathbf{L}^{2}}^{2},
$$

is thus equivalent to the $\Delta^{*}$-supersmoothing of the multiplication operator on $\mathbb{R}^{n}$, $n \geq 3$, with multiplier $V^{1 / 2}$. We also have, by (1.30),

$$
\int_{-\infty}^{\infty}\left\|e^{i t \Delta^{*}} \mathbf{f}\right\|_{\mathbf{L}^{2}(V)}^{2} d t \leq C\|\mathbf{f}\|_{\mathbf{L}^{2}}^{2} \quad \mathbf{f} \in \mathbf{L}^{2} .
$$

Similar results can be stated for the evolution wave equation, which in the case of the Navier operator is the natural to consider.

Consider the following forward initial value problem:

$$
\left\{\begin{array}{l}
\partial_{t t} \mathbf{u}-\Delta^{*} \mathbf{u}=\mathbf{F}(x, t), \quad(x, t) \in \mathbb{R}^{n} \times \mathbb{R}^{+}, n \geq 2, \\
\mathbf{u}(x, 0)=\mathbf{f}(x), \\
\mathbf{u}_{t}(x, 0)=\mathbf{g}(x)
\end{array}\right.
$$


Theorem 1.6. Let $\mathbf{u}(x, t)$ be a solution of (1.33) with $\mathbf{f}=\mathbf{g}=\mathbf{0}$ and, let $V(x)$ be a nonnegative function in $\mathcal{L}^{2, r}\left(\mathbb{R}^{n}\right)$ with $(n-1) / 2<r \leq n / 2$ and $n>2$. Then there exists a positive constant $C$, only depending on $n$, such that the following a priori estimate holds:

$$
\begin{aligned}
& \sup _{x_{0} \in \mathbb{R}^{n}, R>0} \frac{1}{R} \int_{B\left(x_{0}, R\right)} \int_{0}^{\infty}\left|D_{x}^{1 / 2} \mathbf{u}(x, t)\right|^{2} d t d x \\
& \leq C\|V\|_{\mathcal{L}^{2, r}} \int_{0}^{\infty}\|\mathbf{F}(\cdot, t)\|_{\mathbf{L}^{2}\left(V^{-1}\right)}^{2} d t .
\end{aligned}
$$

Theorem 1.7. Let $\mathbf{u}(x, t)$ be a solution of (1.33) with $\mathbf{F}=\mathbf{0}$. If $V(x)$ satisfies the conditions of Theorem 1.6 then there exists a positive constant $C$, only depending on $n$, such that for any $\gamma \in \mathbb{R}$ the following a priori estimates hold:

$$
\begin{array}{r}
\int_{0}^{\infty}\left\|D_{x}^{\gamma} \mathbf{u}(\cdot, t)\right\|_{\mathbf{L}^{2}(V)}^{2} d t \leq C\|V\|_{\mathcal{L}^{2, r}}\left(\left\|D^{\gamma+1 / 2} \mathbf{f}\right\|_{\mathbf{L}^{2}}^{2}+\left\|D^{\gamma-1 / 2} \mathbf{g}\right\|_{\mathbf{L}^{2}}^{2}\right), \\
n>2, \\
\sup _{x_{0} \in \mathbb{R}^{n}, R>0} \frac{1}{R} \int_{B\left(x_{0}, R\right)} \int_{0}^{\infty}\left|D_{x}^{\gamma} \mathbf{u}(x, t)\right|^{2} d t d x \leq C\left(\left\|D^{\gamma} \mathbf{f}\right\|_{\mathbf{L}^{2}}^{2}+\left\|D^{\gamma-1} \mathbf{g}\right\|_{\mathbf{L}^{2}}^{2}\right), \\
n>1 .
\end{array}
$$

Remark 1.8. As above it would be of interest to obtain the above estimates by the multiplier method, this would allow to prove lower estimates (see ([30])).

Acknowledgements. We would like to thank A. Carbery, E. Hernández, F.J. Martin-Reyes and L. Vega for enlightening conversations and for suggesting proper references.

\section{The resolvent operator and the spectral measure}

We introduce the Leray's projection operator I $-\mathcal{R}$, where I denotes the identity matrix of order $n$, and $\mathcal{R}$ is defined via the Fourier transform by

$$
(\mathcal{R} \mathbf{f})(\xi)=\left(\widehat{\mathbf{f}}(\xi) \cdot \frac{\xi}{|\xi|}\right) \frac{\xi}{|\xi|}, \quad \xi \in \mathbb{R}^{n},
$$

with $\mathbf{f} \in L^{2}\left(\mathbb{R}^{n}\right)$ and $\widehat{\mathbf{f}}(\xi)=\left(\widehat{f}_{1}(\xi), \ldots, \widehat{f}_{n}(\xi)\right)$. Observe that $\mathcal{R} \mathbf{f}$ can be written as multiplication by the operator matrix $\left(R_{i} R_{j}\right)$ :

$$
\mathcal{R} \mathbf{f}=-\left(R_{1}\left(\sum_{j=1}^{n} R_{j} f_{j}\right), \ldots, R_{n}\left(\sum_{j=1}^{n} R_{j} f_{j}\right)\right),
$$


where $R_{j}$ for $j=1, \ldots, n$, are the Riesz transforms defined for any $g \in L^{2}\left(\mathbb{R}^{n}\right)$, via the Fourier transform, by

$$
\left(R_{j} g\right) \widehat{\Upsilon}(\xi)=-i \frac{\xi_{j}}{|\xi|} \widehat{g}(\xi) .
$$

The following lemma is easy to prove using the Fourier transform.

Lemma 2.1. The following identities hold for functions $\mathbf{h}$ in the appropriate space:

$$
\begin{aligned}
\mathcal{R}^{2} \mathbf{h} & =\mathcal{R} \mathbf{h}, \\
(\mathrm{I}-\mathcal{R})^{2} \mathbf{h} & =(\mathrm{I}-\mathcal{R}) \mathbf{h}, \\
(\mathrm{I}-\mathcal{R}) \mathcal{R} \mathbf{h} & =\mathcal{R}(\mathrm{I}-\mathcal{R}) \mathbf{h}=0, \\
\Delta \mathcal{R} \mathbf{h} & =\nabla \operatorname{div} \mathbf{h},
\end{aligned}
$$

where $\mathcal{R}$ is defined by (2.1) and I denotes the identity matrix of order $n$.

The following lemma relates resolvent operators of Navier equations and resolvent operators of Helmholtz equations using the Riesz transforms.

Lemma 2.2. Let $z=\gamma+i \varepsilon$ with $\varepsilon \neq 0$ and $\mathbf{f} \in \mathbf{L}^{2}\left(\mathbb{R}^{n}\right)$. The following identity holds:

$$
\left(\Delta^{*}+z \mathrm{I}\right)^{-1} \mathbf{f}=\frac{1}{2 \mu+\lambda}\left(\Delta+\frac{z}{2 \mu+\lambda}\right)^{-1} \mathcal{R} \mathbf{f}+\frac{1}{\mu}\left(\Delta+\frac{z}{\mu}\right)^{-1}(\mathrm{I}-\mathcal{R}) \mathbf{f},
$$

where $\mathcal{R}$ is given by (2.1) and

$$
(\Delta+z)^{-1} \mathbf{f}=\left((\Delta+z)^{-1} f_{1},(\Delta+z)^{-1} f_{2}, \cdots,(\Delta+z)^{-1} f_{n}\right) .
$$

Proof. Given $\mathbf{f} \in \mathbf{L}^{2}\left(\mathbb{R}^{n}\right)$ we consider the resolvent operator $\left(\Delta^{*}+z \mathrm{I}\right)^{-1}$, and we write

$$
\mathbf{u}=\left(\Delta^{*}+z \mathbf{I}\right)^{-1} \mathbf{f},
$$

or equivalently

$$
\left(\Delta^{*}+z \mathrm{I}\right) \mathbf{u}=\mathbf{f} .
$$

Note that applying the projection $\mathcal{R}$ to both sides of this equation and, taking into account that the operators $\mathcal{R}$ and $\Delta \mathrm{I}$ commute, from (2.6) and (2.3), we get the following vectorial equation:

$$
\left(\Delta+z(2 \mu+\lambda)^{-1}\right) \mathcal{R} \mathbf{u}=(2 \mu+\lambda)^{-1} \mathcal{R} \mathbf{f},
$$

and therefore,

$$
\mathcal{R} \mathbf{u}=(2 \mu+\lambda)^{-1}\left(\Delta+z(2 \mu+\lambda)^{-1}\right)^{-1} \mathcal{R} \mathbf{f}
$$


Arguing in a similar way with the operator I $-\mathcal{R}$, from (2.6) and (2.5), we get

$$
\left(\Delta+z \mu^{-1}\right)(\mathrm{I}-\mathcal{R}) \mathbf{u}=\mu^{-1}(\mathrm{I}-\mathcal{R}) \mathbf{f},
$$

and therefore,

$$
(\mathrm{I}-\mathcal{R}) \mathbf{u}=\mu^{-1}\left(\Delta+z \mu^{-1}\right)^{-1}(\mathrm{I}-\mathcal{R}) \mathbf{f} .
$$

Since $\mathbf{u}=\mathcal{R} \mathbf{u}+(\mathrm{I}-\mathcal{R}) \mathbf{u}$, the result follows from (2.8), (2.10) and (2.11).

As a consequence we give a precise description of the spectral measure of the operator $\Delta^{*}$.

Lemma 2.3. If the weak limiting absorption principle holds for the operator $\Delta^{*}$ in a Banach space $\mathbf{B}\left(\mathbb{R}^{n}\right)$, for $\mathbf{f}, \mathbf{g} \in \mathcal{S}\left(\mathbb{R}^{n}\right) \cap \mathbf{B}\left(\mathbb{R}^{n}\right)$, where $\mathcal{S}\left(\mathbb{R}^{n}\right)$ denotes the Schwartz class, we have that

$$
-\int_{\mathbb{R}^{n}} \Delta^{*} \mathbf{f}(x) \mathbf{g}(x) d x=\int_{0}^{\infty} \omega\left\langle d P_{\omega}(\mathbf{f}), \mathbf{g}\right\rangle .
$$

$\left\langle d P_{\omega}(\mathbf{f}), \mathbf{g}\right\rangle$ is given by the density function,

$$
\left\langle d P_{\omega}(\mathbf{f}), \mathbf{g}\right\rangle=\left\langle\frac{1}{\sqrt{\lambda+2 \mu}} \widehat{d \sigma} \sqrt{\frac{\omega}{\lambda+2 \mu}} * \mathcal{R} \mathbf{f}+\frac{1}{\sqrt{\mu}} \widehat{d \sigma} \sqrt{\frac{\omega}{\mu}} *(\mathbf{f}-\mathcal{R} \mathbf{f}), \mathbf{g}\right\rangle \frac{d \omega}{2 \sqrt{\omega}},
$$

where $\langle\cdot, \cdot\rangle$ denotes the scalar product in $\mathbb{C}^{n}, d \sigma_{k}$ denotes the measure on the sphere of radius $k$ induced by the Lebesgue measure of $\mathbb{R}^{n}, \mathcal{R}$ is defined by (2.1) and, $h * \mathbf{f}=\left(h * f_{1}, \ldots, h * f_{n}\right)$.

Proof. By polarization we may reduce to the case $\mathbf{g}=\mathbf{f}$.

Recall Stone's formula for the distribution function of the spectral projection measures of any selfadjoint extension of $-\Delta^{*}$. Let $[a, b] \subset(0, \infty)$, then

$$
\begin{aligned}
& \langle(P(b)-P(a)) \mathbf{f}, \mathbf{f}\rangle \\
& =\lim _{\delta \rightarrow 0^{+}} \lim _{\epsilon \rightarrow 0^{+}} \frac{1}{2 \pi i} \int_{a+\delta}^{b+\delta}\left\langle\left(\left(-\Delta^{*}-(\omega+i \epsilon) \mathrm{I}\right)^{-1}-\left(-\Delta^{*}-(\omega-i \epsilon) \mathrm{I}\right)^{-1}\right) \mathbf{f}, \mathbf{f}\right\rangle d \omega .
\end{aligned}
$$

From (1.14), we have that

$$
\left|\left\langle\left(-\Delta^{*}-(\omega \pm i \epsilon) \mathrm{I}\right)^{-1} \mathbf{f}, \mathbf{f}\right)\right| \leq c\|\mathbf{f}\|_{\mathbf{B}}^{2},
$$

and hence, using bounded convergence and the continuity of the spectrum,

$$
\begin{aligned}
\langle(P(b) & -P(a)) \mathbf{f}, \mathbf{f}\rangle \\
& =\frac{1}{2 \pi i} \int_{a}^{b} \lim _{\epsilon \rightarrow 0^{+}}\left\langle\left(\left(-\Delta^{*}-(\omega+i \epsilon) \mathrm{I}\right)^{-1}-\left(-\Delta^{*}-(\omega-i \epsilon) \mathrm{I}\right)^{-1}\right) \mathbf{f}, \mathbf{f}\right\rangle d \omega .
\end{aligned}
$$


Then, using (2.7), together with the following distributional identity for $k \in \mathbb{R}$

$$
\begin{aligned}
& \lim _{\epsilon \rightarrow 0^{+}}(-\Delta-k \pm i \epsilon)^{-1} h \\
& =p . v \cdot \int_{\mathbb{R}^{n}} \frac{1}{|\xi|^{2}-k} \widehat{h}(\xi) e^{i x \cdot \xi} d \xi \mp \frac{i \pi}{2 \sqrt{k}} \chi_{\{k>0\}} \widehat{d \sigma_{\sqrt{k}}} * h(x),
\end{aligned}
$$

where $\chi_{\{k>0\}}$ takes the value 1 if $k>0$ and 0 otherwise, we get that

$$
\begin{aligned}
\langle(P(b) & -P(a)) \mathbf{f}, \mathbf{f}\rangle \\
& =\int_{a}^{b}\left\langle\left(\frac{1}{\sqrt{\lambda+2 \mu}} \widehat{d \sigma} \widehat{\sqrt{\frac{\omega}{\lambda+2 \mu}}} \mathrm{I} * \mathcal{R}+\frac{1}{\sqrt{\mu}} \widehat{d \sigma} \sqrt{\frac{\omega}{\mu}} \mathrm{I} *(\mathrm{I}-\mathcal{R})\right) \mathbf{f}, \mathbf{f}\right\rangle \frac{d \omega}{2 \sqrt{\omega}} .
\end{aligned}
$$

Now we can reach the point $a=0$ by monotone convergence, since the quantity inside the previous integral is nonnegative.

Using the spectral operational calculus, the above description of the spectral resolution allows us to define extensions of the operators given by spectral functions $f(\omega)$ as, for instance, solutions of the initial value problem of the wave equation (see Subsection 3.2)

$$
\left(\frac{\partial^{2}}{\partial t^{2}}-\Delta^{*}\right) \mathbf{u}=\mathbf{0},
$$

or of the Schrödinger equation. This formula reduces the operational calculus to the study of Stein-Tomas operator given by convolution with $\frac{1}{\sqrt{k}} \widehat{d \sigma_{\sqrt{k}}}$. Boundedness properties of this operator suggest the appropriate domains for selfadjoint extensions of the operator $\Delta^{*}$ itself.

\section{Proofs}

\subsection{Spectral Navier equation}

It is natural, after Lemma 2.2, to study the behavior of singular integrals in the spaces considered in the statements of the theorems. We start by recalling some definitions.

Definition 3.1. Let $w$ be a measurable nonnegative function in $L_{\ell \mathrm{oc}}^{1}\left(\mathbb{R}^{n}\right)$. For $1<$ $p<\infty$, we will say that $w$ is a weight in the $A_{p}$ class, and we will write $w \in A_{p}$, if and only if for any cube $Q$ in $\mathbb{R}^{n}$ we have that

$$
\left(\frac{1}{|Q|} \int_{Q} w\right)\left(\frac{1}{|Q|} \int_{Q} w^{-1 /(p-1)}\right)^{p-1} \leq C
$$

with $C$ a constant independent of $Q$. 
We will say that $w$ is a weight in the $A_{1}$ class, and we will write $w \in A_{1}$, if and only if $M w(x) \leq C w(x)$, for almost all $x \in \mathbb{R}^{n}$, where $M$ denotes the usual Hardy-Littlewood maximal operator, defined for any $g \in L_{\ell o c}^{1}\left(\mathbb{R}^{n}\right)$ by

$$
M g(x)=\sup _{r>0} \frac{1}{B_{r}} \int_{B_{r}}|g(x-y)| d y,
$$

where $B_{r}$ is the Euclidean ball centered at the origin with radius $r$.

For $1 \leq p<\infty$, the smallest constant $C$ satisfying the corresponding previous condition is called the $A_{p}$ constant for $w$.

From this definition, it is easy to prove that $A_{p} \subset A_{q}$ if $p<q$. For more information about the $A_{p}$ class see [11].

Lemma 3.2. Let $1<p<\infty$ and $w \in A_{p}$. For $j=1, \ldots, n$, the following inequalities hold:

$$
\begin{aligned}
\left\|R_{j} g\right\|_{X} & \leq c\|g\|_{X}, \\
\left\|R_{j} g\right\|_{L^{p}} & \leq c\|g\|_{L^{p}}, \\
\left\|R_{j} g\right\|_{L^{p}(w)} & \leq c_{w}\|g\|_{L^{p}(w)},
\end{aligned}
$$

where $R_{j}$ are the Riesz transforms, $c$ is a constant independent of $g$, and $c_{w}$ is a constant depending on the $A_{p}$ constant for $w$ but independent of $g$.

Proof. This lemma gathers several known estimates. The proofs of (3.2) and (3.3) can be found in [5] and [9] respectively.

Let us prove (3.1) (see [21], where a very general class of spaces is considered).

For $k \in \mathbb{Z}$, let $\chi_{k}=\chi_{C_{k}}$, where $C_{k}$ is given by (1.17). With this notation, since $R_{j}$ is a linear operator, we can write

$$
g=\sum_{k \in \mathbb{Z}} g \chi_{k} \quad \text { and } \quad R_{j} g=\sum_{k \in \mathbb{Z}} R_{j}\left(g \chi_{k}\right),
$$

and therefore,

$$
\begin{aligned}
\left\|R_{j} g\right\|_{X} & =\sum_{m \in \mathbb{Z}} 2^{(m+1) / 2}\left\|\chi_{m} R_{j} g\right\|_{L^{2}} \\
& \leq \sum_{m \in \mathbb{Z}} 2^{(m+1) / 2} \sum_{k \in \mathbb{Z}}\left\|\chi_{m} R_{j}\left(g \chi_{k}\right)\right\|_{L^{2}} .
\end{aligned}
$$

On the other hand, taking into account that

$$
R_{j} g(x)=c_{n} \text { p.v. } \int_{\mathbb{R}^{n}} \frac{x_{j}-y_{j}}{|x-y|^{n+1}} g(y) d y,
$$

where $c_{n}=\Gamma((n+1) / 2) \pi^{-(n+1) / 2}$, for $m \in \mathbb{Z}$ fixed, using the Cauchy-Schwarz inequality, it is easy to prove that if $x \in C_{m}$, then

$$
\left|R_{j}\left(g \chi_{k}\right)(x)\right| \leq \begin{cases}c 2^{-m n} 2^{k n / 2}\left\|g \chi_{k}\right\|_{L^{2}} & \text { if } k<m-1, \\ c 2^{-k n / 2}\left\|g \chi_{k}\right\|_{L^{2}} & \text { if } k>m+1\end{cases}
$$


Thus, if we split the second sum in (3.4) into three parts, for $k<m-1$, for $m-1 \leq k \leq m+1$ and for $k>m+1$, we have that

$$
\begin{aligned}
\left\|R_{j} g\right\|_{X} \leq & c \sum_{k \in \mathbb{Z}} 2^{k n / 2}\left\|g \chi_{k}\right\|_{L^{2}} \sum_{m>k+1} 2^{-m(n-1) / 2} \\
& +\sum_{m \in \mathbb{Z}} 2^{(m+1) / 2} \sum_{k=m-1}^{m+1}\left\|R_{j}\left(g \chi_{k}\right)\right\|_{L^{2}} \\
& +c \sum_{k \in \mathbb{Z}} 2^{-k n / 2}\left\|g \chi_{k}\right\|_{L^{2}} \sum_{m<k-1} 2^{m(n+1) / 2} .
\end{aligned}
$$

The result follows from here using (3.2) with $p=2$.

Given a nonnegative function in a Morrey-Campanato space, the following result (see [7, Lemma 1]) gives a method to construct a majorizing function in the $A_{1}$ class, and consequently in the $A_{2}$ class, within the same Morrey-Campanato space.

Lemma 3.3. Let $V$ be a nonnegative function in $\mathcal{L}^{\alpha, r}\left(\mathbb{R}^{n}\right)$, with $0<\alpha<n$ and $1<r \leq n / \alpha$. If $r_{1}$ is such that $1<r_{1}<r$, then $W=\left(M V^{r_{1}}\right)^{1 / r_{1}} \in A_{1} \cap \mathcal{L}^{\alpha, r}\left(\mathbb{R}^{n}\right)$, where $M$ denotes the usual Hardy-Littlewood maximal operator.

Furthermore, there exists a constant $c>0$ independent of $V$, such that the $A_{1}$ constant for $W$ is less than $c$ and

$$
\|W\|_{\mathcal{L}^{\alpha, r}} \leq c\|V\|_{\mathcal{L}^{\alpha, r}}
$$

Remark 3.4. Observe that $V(x) \leq W(x)$ for almost all $x \in \mathbb{R}^{n}$.

In order to construct a majorizing function in the $A_{1}$ class for a nonnegative function in a $D_{p}\left(\mathbb{R}^{n}\right)$ space, which is in the same $D_{p}\left(\mathbb{R}^{n}\right)$ space, we will need the following results, that can be found in [12] and [14] respectively (see [12, Lemma 5.1 in Chapter IV] and [14, Corollary 2.1]).

Lemma 3.5 (Rubio de Francia algorithm). Let $E$ be a normed space of complex functions with norm $\|\cdot\|_{E}$, and let $S$ be a sublinear operator (i.e. $|S(a F+b G)| \leq$ $|a||S(F)|+|b||S(G)|, \forall a, b \in \mathbb{C}, \forall F, G \in E)$ bounded in $E$ with norm $\|S\|$. If $S F \geq 0$ for all $F \in E$, then for all nonnegative $V$ in $E$, the function

$$
W=\sum_{j=0}^{\infty}(2\|S\|)^{-j} S^{j} V
$$

is also a nonnegative function in $E$ such that

(i) $V(x) \leq W(x)$ for almost all $x \in \mathbb{R}^{n}$,

(ii) $\|W\|_{E} \leq 2\|V\|_{E}$,

(iii) $S W(x) \leq C W(x)$ for almost all $x \in \mathbb{R}^{n}$, with $C=2\|S\|$. 
Lemma 3.6. If $1<p \leq \infty$, then the usual Hardy-Littlewood maximal operator is bounded on $D_{p}\left(\mathbb{R}^{n}\right)$.

Proof. (See [14] for general Herz spaces).

Consider $1<p<\infty$ (the proof for $p=\infty$ is easier). Arguing as in the proof of (3.1), taking into account that $M$ is a sublinear operator, we have that

$$
\|M g\|_{D_{p}} \leq \sum_{m \in \mathbb{Z}} 2^{m(p-n) / p} \sum_{k \in \mathbb{Z}}\left\|\chi_{m} M\left(g \chi_{k}\right)\right\|_{L^{p}}
$$

On the other hand, for $m \in \mathbb{Z}$ fixed, using the Holder inequality, it is easy to prove that if $x \in C_{m}$, then

$$
\left|M\left(g \chi_{k}\right)(x)\right| \leq \begin{cases}c 2^{-m n} 2^{k n(p-1) / p}\left\|g \chi_{k}\right\|_{L^{p}} & \text { if } k<m-1, \\ c 2^{-k n / p}\left\|g \chi_{k}\right\|_{L^{p}} & \text { if } k>m+1\end{cases}
$$

Thus, if we split the second sum in (3.6) into three parts, for $k<m-1$, for $m-1 \leq k \leq m+1$ and for $k>m+1$, we have that

$$
\begin{aligned}
\|M g\|_{D_{p}} \leq & c \sum_{k \in \mathbb{Z}} 2^{k n(p-1) / p}\left\|g \chi_{k}\right\|_{L^{p}} \sum_{m>k+1} 2^{-m(n-1)} \\
& +\sum_{m \in \mathbb{Z}} 2^{m(p-n) / p} \sum_{k=m-1}^{m+1}\left\|M\left(g \chi_{k}\right)\right\|_{L^{p}} \\
& +c \sum_{k \in \mathbb{Z}} 2^{-k n / p}\left\|g \chi_{k}\right\|_{L^{p}} \sum_{m<k-1} 2^{m} .
\end{aligned}
$$

The result follows from here using the fact that $M$ is a bounded operator on $L^{p}\left(\mathbb{R}^{n}\right)$ for $1<p \leq \infty$.

Remark 3.7. From Lemma 3.6 we know that if $1<p \leq \infty$ then, the usual Hardy-Littlewood maximal operator, $M$, is bounded on $D_{p}\left(\mathbb{R}^{n}\right)$. Thus, we can apply Lemma 3.5 to $E=D_{p}\left(\mathbb{R}^{n}\right)$ with $1<p \leq \infty$ and $S=M$, to conclude that given a nonnegative function $V$ in $D_{p}\left(\mathbb{R}^{n}\right)$ there exists a nonnegative $W$ in $D_{p}\left(\mathbb{R}^{n}\right) \cap A_{1}$, and therefore in $D_{p}\left(\mathbb{R}^{n}\right) \cap A_{2}$, such that $V(x) \leq W(x)$ for almost all $x \in \mathbb{R}^{n}$.

Furthermore, there exists a constant $c>0$ independent of $V$, such that the $A_{1}$ constant for $W$ is less than $c$ and

$$
\|W\|_{D_{p}} \leq c\|V\|_{D_{p}}, \quad 1<p \leq \infty .
$$

Moreover, since the Maximal function of a radial function is also a radial function, if $V$ is a radial function, then $W$ is also a radial function. 
We will use the following estimates for the resolvent of the Laplacian:

Theorem 3.8. Let $z=\gamma+i \varepsilon$ with $\varepsilon \neq 0, \frac{1}{p}+\frac{1}{q}=1$ with $\frac{2}{n+1} \leq \frac{1}{p}-\frac{1}{q} \leq \frac{2}{n}$ if $n>2$, or $\frac{2}{3} \leq \frac{1}{p}-\frac{1}{q}<1$ if $n=2$, and let $V_{1}$ be a nonnegative function in $\mathcal{L}^{2, r}\left(\mathbb{R}^{n}\right)$ with $(n-1) / 2<r \leq n / 2$ and $n>2$, and $V_{2} \in \mathcal{T}\left(\mathbb{R}^{n}\right)$. If $g \in L^{2}\left(\mathbb{R}^{n}\right)$ then, there exists a constant $c>0$ independent of $z, g, V_{1}$ and $V_{2}$ such that the following a priori estimates hold:

$$
\begin{aligned}
\left\|(\Delta+z)^{-1} g\right\|_{X^{*}} & \leq c|z|^{-1 / 2}\|g\|_{X}, \\
\left\|(\Delta+z)^{-1} g\right\|_{L^{q}} & \leq c|z|^{\frac{n}{2}\left(\frac{1}{p}-\frac{1}{q}\right)-1}\|g\|_{L^{p}}, \\
\left\|(\Delta+z)^{-1} g\right\|_{L^{2}\left(V_{1}\right)} & \leq c\left\|V_{1}\right\|_{\mathcal{L}^{2, r}}\|g\|_{L^{2}\left(V_{1}^{-1}\right)}, \\
\left\|(\Delta+z)^{-1} g\right\|_{L^{2}\left(V_{2}\right)} & \leq c|z|^{-1 / 2} \mid\left\|V_{2}\right\|\|\| g \|_{L^{2}\left(V_{2}^{-1}\right)}
\end{aligned}
$$

Besides, the weak limiting absorption principle holds for $\Delta$ in the spaces $X\left(\mathbb{R}^{n}\right)$, $L^{q}\left(\mathbb{R}^{n}\right), L^{2}\left(V_{1}\right)$ and $L^{2}\left(V_{2}\right)$ for the Sommerfeld radiation condition (1.12).

Theorem 3.9. Let $p$ such that $\frac{1}{n+1} \leq \frac{1}{p}-\frac{1}{2} \leq \frac{1}{n}$, and let $V_{1}$ be a nonnegative function in $\mathcal{L}^{2, r}\left(\mathbb{R}^{n}\right)$ with $(n-1) / 2<r \leq n / 2$ and $n>2$, and $V_{2} \in \mathcal{T}\left(\mathbb{R}^{n}\right)$. If $g \in \mathcal{C}_{0}^{\infty}\left(\mathbb{R}^{n}\right)$ and we consider the unique solution $v$ of equation (1.11) satisfying (1.12), then, there exists a constant $c>0$ independent of $k, g, V_{1}$ and $V_{2}$ such that the following a priori estimates hold:

$$
\begin{aligned}
\|\nabla v\|_{X^{*}} & \leq c\|g\|_{X}, \\
\sup _{x_{0}, R>0} \frac{1}{R} \int_{B\left(x_{0}, R\right)}|v(x)|^{2} d x & \leq c k^{2 n\left(\frac{1}{p}-\frac{1}{2}\right)-3}\|g\|_{L^{p}}^{2}, \\
\sup _{x_{0}, R>0} \frac{1}{R} \int_{B\left(x_{0}, R\right)}\left|D^{1 / 2} v(x)\right|^{2} d x & \leq c\left\|V_{1}\right\|_{\mathcal{L}^{2, r} \|}\|g\|_{L^{2}\left(V_{1}^{-1}\right)}^{2}, \\
\|\nabla v\|_{L^{2}\left(V_{2}\right)} & \leq c\|\| V_{2}\|\|\|\|_{L^{2}\left(V_{2}^{-1}\right)},
\end{aligned}
$$

where $D^{1 / 2} v$ is defined via the Fourier transform as $\left(D^{1 / 2} v\right)^{-}(\xi)=|\xi|^{1 / 2} \widehat{v}(\xi), \xi \in$ $\mathbb{R}^{n}$.

The results given in (3.7) and (3.11) can be found essentially in [17] (see [17, Lemma 2.4]). For a complete proof see [24] (see also [1] and [2]). The result given in (3.8) can be found in [18] and, (3.9) and (3.13) in [28]. The result given in (3.12) can be found in [27] (see also [23]). Finally, the results given in (3.10) and (3.14) can be found in [4].

The estimates (3.10) and (3.14) are a generalization of (3.7) and (3.11) respectively. Estimates related to (3.9) can be found in [8].

Remark 3.10. From (1.19), the estimates (3.10) and (3.14) also hold if we replace $\left\|\left|V_{2}\right|\right\|$ with $\left\|V_{2}\right\|_{D_{\tilde{r}}}$, with $\tilde{r}>2$ and $V_{2}$ being a radial function. 
Proof of Theorem 1.1. We start with the proof of (1.20). Given $\mathbf{f} \in \mathbf{L}^{2}\left(\mathbb{R}^{n}\right)$, from (2.7), it is enough to prove the result for the operator

$$
(\Delta+z)^{-1} \mathcal{R} \mathbf{f}
$$

Using (2.2), the triangular inequality, (3.7) and (3.1) we have that

$$
\begin{aligned}
\left\|(\Delta+z)^{-1} \mathcal{R} \mathbf{f}\right\|_{\mathbf{X}^{*}} & \leq \sum_{i=1}^{n}\left\|(\Delta+z)^{-1} R_{i}\left(\sum_{j=1}^{n} R_{j} f_{j}\right)\right\|_{X^{*}} \\
& \leq c|z|^{-1 / 2}\left\|R_{i}\left(\sum_{j=1}^{n} R_{j} f_{j}\right)\right\|_{X} \\
& \leq c|z|^{-1 / 2}\|\mathbf{f}\|_{\mathbf{X}} .
\end{aligned}
$$

We omit the proof of (1.21) since it is similar to the proof of (1.20), but using (3.8) and (3.2) instead of (3.7) and (3.1). The proofs of (1.22) and (1.23) require an additional argument because in order to use (3.3) for $p=2$ we need a weight in the $A_{2}$ class.

As before, it is enough to prove (1.22) and (1.23) for the operator given in (3.15).

Let $W_{1}=\left(M V_{1}^{r_{1}}\right)^{1 / r_{1}}$, with $1<r_{1}<r$. Observe that under the conditions of the theorem, from Lemma 3.3 we know that $W_{1} \in A_{2} \cap \mathcal{L}^{2, r}\left(\mathbb{R}^{n}\right)$.

Using (2.2) and the triangular inequality, since $V_{1}(x) \leq W_{1}(x)$, we have that

$$
\begin{aligned}
\left\|(\Delta+z)^{-1} \mathcal{R} \mathbf{f}\right\|_{\mathbf{L}^{2}\left(V_{1}\right)} & \leq \sum_{i=1}^{n}\left\|(\Delta+z)^{-1} R_{i}\left(\sum_{j=1}^{n} R_{j} f_{j}\right)\right\|_{L^{2}\left(V_{1}\right)} \\
& \leq \sum_{i=1}^{n}\left\|(\Delta+z)^{-1} R_{i}\left(\sum_{j=1}^{n} R_{j} f_{j}\right)\right\|_{L^{2}\left(W_{1}\right)} .
\end{aligned}
$$

From here, applying (3.9), (3.5) with $\alpha=2$, and (3.3) with $p=2$ we have that

$$
\begin{aligned}
\left\|(\Delta+z)^{-1} \mathcal{R} \mathbf{f}\right\|_{\mathbf{L}^{2}\left(V_{1}\right)} & \leq c\left\|W_{1}\right\|_{\mathcal{L}^{2, r}} \sum_{i=1}^{n}\left\|R_{i}\left(\sum_{j=1}^{n} R_{j} f_{j}\right)\right\|_{L^{2}\left(W_{1}^{-1}\right)} \\
& \leq c_{W_{1}}\left\|V_{1}\right\|_{\mathcal{L}^{2, r}}\|\mathbf{f}\|_{\mathbf{L}^{2}\left(W_{1}^{-1}\right)} \\
& \leq c_{W_{1}}\left\|V_{1}\right\|_{\mathcal{L}^{2, r},\|\mathbf{f}\|_{\mathbf{L}^{2}\left(V_{1}^{-1}\right)}}
\end{aligned}
$$

Since from Lemma 3.3 we know that $c_{W_{1}}$ is less than a constant independent of $V_{1}$, estimate (1.22) follows from here.

Estimate (1.23) can be obtained in a similar way taking into account that, as we explained in Remark 3.7, given $V_{2}$ satisfying the hypotheses of the theorem, 
we can construct a majorizing function $W_{2} \in D_{p}\left(\mathbb{R}^{n}\right) \cap A_{2}$ which properties are similar to the properties of $W_{1}$. In this case, instead of (3.9) we have to use (3.10) and Remark 3.10.

The existence of the weak limit follows in a standard way (see [1]).

To finish the proof, it remains to show that for $\mathbf{f} \in \mathbf{C}_{0}^{\infty}\left(\mathbb{R}^{n}\right)$, the solution $\mathbf{R}\left(\omega^{2}+i 0\right) \mathbf{f}$ defined by (1.15) satisfies the Kupradze radiation conditions, given in (1.6) and (1.7).

Consider $\mathbf{f} \in \mathbf{C}_{0}^{\infty}\left(\mathbb{R}^{n}\right)$, and write $\mathbf{u}=\mathbf{R}\left(\omega^{2}+i 0\right) \mathbf{f}$. From (2.7), and using the weak limiting absorption principle for the Helmholtz equation, we can write that

$$
\mathbf{u}=\frac{1}{2 \mu+\lambda} R\left(k_{p}^{2}+i 0\right) \mathcal{R} \mathbf{f}+\frac{1}{\mu} R\left(k_{s}^{2}+i 0\right)(\mathrm{I}-\mathcal{R}) \mathbf{f},
$$

where $k_{p}$ and $k_{s}$ are defined by (1.3) and $R\left(k^{2}+i 0\right)$ by

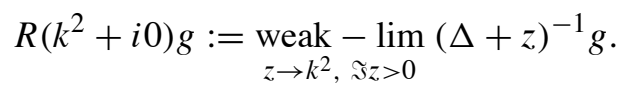

As a consequence, since the operators $R\left(k^{2}+i 0\right)$ and $R_{j}, j=1, \ldots, n$, commute with the operators $\partial^{\alpha}, \alpha \in \mathbb{R}^{n}$, we know that $\mathbf{u} \in \mathbf{C}^{\infty}\left(\mathbb{R}^{n}\right)$.

On one hand, using the Fourier transform, it is easy to prove that $\nabla \operatorname{div} \mathcal{R} \mathbf{h}=$ $\nabla \operatorname{div} \mathbf{h}$, for $\mathbf{h}$ in the appropriate space. Using this identity, since the operators $R\left(k^{2}+i 0\right)$ commute with the operators $\partial^{\alpha}, \alpha \in \mathbb{R}^{n}$, from (3.16), we obtain that

$$
\mathbf{u}_{p}=-\frac{1}{k_{p}^{2}} \nabla \operatorname{div} \mathbf{u}=-\frac{1}{\omega^{2}} R\left(k_{p}^{2}+i 0\right) \nabla \operatorname{div} \mathbf{f} .
$$

For $\mathbf{f} \in \mathbf{C}_{0}^{\infty}\left(\mathbb{R}^{n}\right)$, we have that $\nabla \operatorname{div} \mathbf{f} \in \mathbf{C}_{0}^{\infty}\left(\mathbb{R}^{n}\right)$, and therefore, by Theorem 3.8 we know that for $j=1, \ldots, n, R\left(k_{p}^{2}+i 0\right) \partial_{j} \operatorname{div} \mathbf{f}$ satisfies the corresponding Sommerfeld outgoing radiation condition, that is

$$
\left(\partial_{r}-i k_{p}\right) R\left(k_{p}^{2}+i 0\right) \partial_{j} \operatorname{div} \mathbf{f}=o\left(r^{-(n-1) / 2}\right) .
$$

Hence, $\mathbf{u}$ satisfies the condition (1.6).

On the other hand, since $\mathbf{u}$ satisfies the equation (1.8), we have that

$$
\mathbf{u}=\frac{1}{\omega^{2}} \mathbf{f}-\frac{\mu}{\omega^{2}} \Delta \mathrm{I} \mathbf{u}-\frac{(\lambda+\mu)}{\omega^{2}} \nabla \operatorname{div} \mathbf{u} .
$$

Using this identity, (1.3) and (2.6), we can write that

$$
\begin{aligned}
\mathbf{u}_{s} & =\mathbf{u}-\mathbf{u}_{p}=\mathbf{u}-\frac{1}{k_{p}^{2}} \nabla \operatorname{div} \mathbf{u} \\
& =\frac{1}{\omega^{2}} \mathbf{f}-\frac{\mu}{\omega^{2}} \Delta \mathrm{I} \mathbf{u}+\frac{\mu}{\omega^{2}} \nabla \operatorname{div} \mathbf{u} \\
& =\frac{1}{\omega^{2}} \mathbf{f}+\frac{\mu}{\omega^{2}} \Delta \mathrm{I}(\mathcal{R}-\mathrm{I}) \mathbf{u} .
\end{aligned}
$$


From here, using the identities (2.7), (2.5) and (2.4), we have that

$$
\begin{aligned}
\mathbf{u}_{s} & =\frac{1}{\omega^{2}} \mathbf{f}+\frac{1}{\omega^{2}} R\left(k_{s}^{2}+i 0\right) \Delta \mathrm{I}(\mathcal{R}-\mathrm{I}) \mathbf{f} \\
& =\frac{1}{\omega^{2}} \mathbf{f}+\frac{1}{\omega^{2}} R\left(k_{s}^{2}+i 0\right)(\nabla \operatorname{div} \mathbf{f}-\Delta \mathrm{I} \mathbf{f}) .
\end{aligned}
$$

For $\mathbf{f} \in \mathbf{C}_{0}^{\infty}\left(\mathbb{R}^{n}\right)$, we have that $\nabla \operatorname{div} \mathbf{f}-\Delta$ If $\in \mathbf{C}_{0}^{\infty}\left(\mathbb{R}^{n}\right)$. From here and from (3.18) we conclude that $\mathbf{u}$ satisfies condition (1.7) arguing as we did for the condition (1.6).

Proof of Theorem 1.2. The proofs of (1.24) and (1.25) are similar to the proof of (1.20), but using (3.11) and (3.12) instead of (3.7) respectively.

Finally, the proofs of (1.26) and (1.27) are similar to the proofs of (1.22) and (1.23), but using (3.13) instead of (3.9) and (3.14) instead of (3.10) respectively.

Remark 3.11. We leave as an open question the extension of the results given in (3.10) and (3.14) for the Helmholtz equation to the Navier equation. This is due to the fact that (in the present work) given a radial nonnegative function with bounded everywhere X-rays transform we need a majorizing function in the $A_{2}$ class with bounded everywhere X-rays transform.

\subsection{Elasticity equation}

Let us start by recalling that for a given nonnegative function $V \in \mathcal{L}^{2, r}\left(\mathbb{R}^{n}\right)$, with $(n-1) / 2<r \leq n / 2$, and $n>2$, by taking the limiting absorption principle in the sense of (3.9), if $\mathbf{f} \in \mathbf{L}^{2}\left(\mathbb{R}^{n}\right) \cap \mathbf{L}^{2}\left(V^{-1}\right)$, for appropriate spectral functions $h$, we can use the functional calculus to write that

$$
h\left(-\Delta^{*}\right) \mathbf{f}=\int_{0}^{\infty} h(\omega) d P_{\omega}(\mathbf{f})(x),
$$

where the projection operator is given by

$$
d P_{\omega}(\mathbf{f})=\frac{1}{2 \sqrt{\lambda+2 \mu} \sqrt{\omega}}\left(\widehat{d \sigma} \frac{1}{\sqrt{\frac{\omega}{\lambda+2 \mu}}} * \mathcal{R} \mathbf{f}\right)+\frac{1}{2 \sqrt{\mu} \sqrt{\omega}}\left(\widehat{d \sigma} \sqrt{\frac{\omega}{\mu}} *(\mathrm{I}-\mathcal{R}) \mathbf{f}\right) .
$$

In particular, the solution of problem (1.33) with $\mathbf{F}=\mathbf{g}=0$ is given by the evolution operator

$$
\mathbf{S}_{1}(t) \mathbf{f}=\cos \left(t \sqrt{-\Delta^{*}}\right) \mathbf{f}=\int_{0}^{\infty} \cos (t \sqrt{\omega}) d P_{\omega}(\mathbf{f}),
$$

and with $\mathbf{F}=\mathbf{f}=0$ by

$$
\mathbf{S}_{2}(t) \mathbf{g}=\frac{\sin \left(t \sqrt{-\Delta^{*}}\right)}{\sqrt{-\Delta^{*}}} \mathbf{g}=\int_{0}^{\infty} \frac{\sin (t \sqrt{\omega})}{\sqrt{\omega}} d P_{\omega}(\mathbf{g})
$$


Theorem 1.7 follows from the expression we obtained for the spectral measure (only the control of the imaginary part of the resolvent is needed). We will use the following weighted extension theorem for the Fourier transform and also the endpoint trace lemma.

Lemma 3.12 (See [26]). Let $\mathbb{S}_{s}^{n-1}$ be the sphere of radius $s$ in $\mathbb{R}^{n}$, and let $V$ be a nonnegative function in $V \in \mathcal{L}^{2, r}\left(\mathbb{R}^{n}\right)$ with $(n-1) / 2<r \leq n / 2$ and $n>2$. Then, there exists a positive constant $C$ only depending on $n$ such that

$$
\left\|\widehat{g d \sigma_{s}}\right\|_{\mathbf{L}^{2}(V)} \leq C s^{1 / 2}\|V\|_{\mathcal{L}^{2, r}}\|g\|_{\mathbf{L}^{2}\left(\mathbb{S}_{s}^{n-1}\right)} .
$$

Lemma 3.13 (See [2]). Let $\mathbb{S}_{s}^{n-1}$ be the sphere of radius $s$ in $\mathbb{R}^{n}$. Then, there exists a positive constant $C$ only depending on $n$ such that

$$
\sup _{x_{0} \in \mathbb{R}^{n}, R>0} \frac{1}{R} \int_{B\left(x_{0}, R\right)}\left|\int_{\mathbb{S}_{s}^{n-1}} e^{i x \cdot \xi} f(\xi) d \sigma_{s}(\xi)\right|^{2} d x \leq C \int_{\mathbb{S}_{s}^{n-1}}|f(\xi)|^{2} d \sigma_{s}(\xi) .
$$

Proof of Theorem 1.7. Assume $g=0$. It is enough to prove (1.35), from (3.20) and (3.19), for the following operator:

$$
\begin{aligned}
\tilde{\mathbf{S}}_{1}(t) \mathbf{f} & =\int_{0}^{\infty} e^{i t \sqrt{\omega}}\left(\widehat{d \sigma}_{\sqrt{\omega}} * \mathcal{R} \mathbf{f}\right) \frac{d \omega}{2 \sqrt{\omega}} \\
& =\int_{0}^{\infty} e^{i t \omega}\left(\widehat{d \sigma}_{\omega} * \mathcal{R} \mathbf{f}\right) d \omega \\
& =\left(\chi_{(0, \infty)}(\cdot)\left(\widehat{d \sigma}_{(\cdot)} * \mathcal{R} \mathbf{f}\right)\right) \widehat{(}(-t) .
\end{aligned}
$$

Taking into account that

$$
D_{x}^{\gamma}\left(\widehat{d \sigma_{\omega}} * \mathbf{f}\right)=\omega^{\gamma}\left(\widehat{d \sigma_{\omega}} * \mathbf{f}\right)
$$

we have that

$$
D_{x}^{\gamma} \tilde{\mathbf{S}}_{1}(t) \mathbf{f}(x)=\left((\cdot)^{\gamma} \chi_{(0, \infty)}(\cdot)\left(\widehat{d \sigma}_{(\cdot)} * \mathcal{R} \mathbf{f}\right)(x) \widehat{)}(-t) .\right.
$$

Therefore, using Fubini, Plancherel's identity and (3.22) we can write

$$
\begin{aligned}
\int_{0}^{\infty}\left\|D_{x}^{\gamma} \tilde{\mathbf{S}}_{1}(t) \mathbf{f}\right\|_{\mathbf{L}^{2}(V)}^{2} d t & =\left\|\left(\int_{0}^{\infty}\left|t^{\gamma}\left(\widehat{d \sigma}_{t} * \mathcal{R} \mathbf{f}\right)\right|^{2} d t\right)^{1 / 2}\right\|_{L^{2}(V)}^{2} \\
& =\int_{0}^{\infty} t^{2 \gamma}\left\|\left(\widehat{\mathcal{R} \mathbf{f}} d \sigma_{t}\right) \widehat{(}(-x)\right\|_{\mathbf{L}^{2}(V)}^{2} d t \\
& \leq C\|V\|_{\mathcal{L}^{2, r}} \int_{0}^{\infty} t^{2 \gamma+1} \int_{|\xi|=t}|\widehat{\mathcal{R} \mathbf{f}}(\xi)|^{2} d \sigma_{t}(\xi) d t \\
& =C\|V\|_{\mathcal{L}^{2, r}} \int_{0}^{\infty} \int_{|\xi|=t}\left|\left(D^{\gamma+1 / 2} \mathcal{R} \mathbf{f}\right)(\xi)\right|^{2} d \sigma_{t}(\xi) d t .
\end{aligned}
$$


Using polar coordinates, the fact that the operator $\mathcal{R}$ commutes with the operators $D^{\gamma}, \gamma \in \mathbb{R}$, Plancherel's identity, (2.2) and (3.2) for $p=2$, we get

$$
\begin{aligned}
\int_{0}^{\infty}\left\|D_{x}^{\gamma} \tilde{\mathbf{S}}_{1}(t) \mathbf{f}\right\|_{\mathbf{L}^{2}(V)}^{2} d t & \leq C\|V\|_{\mathcal{L}^{2, r}} \int_{\mathbb{R}^{n}} \mid\left(\left.\mathcal{R} D^{\gamma+1 / 2} \widehat{\mathbf{f})(\xi)}\right|^{2} d \xi\right. \\
& \leq C\|V\|_{\mathcal{L}^{2, r}}\left\|D^{\gamma+1 / 2} \mathbf{f}\right\|_{\mathbf{L}^{2}}^{2} .
\end{aligned}
$$

Estimate (1.36) can be proved in a similar way, but using (3.23) instead of (3.22).

We omit the proof of Theorem 1.7 in the case $f=0$ because, from (3.21) and (3.19), it is similar to the above case but using the operator

$$
\tilde{\mathbf{S}}_{2}(t) \mathbf{g}=\int_{0}^{\infty} e^{i t \sqrt{\omega}}\left(\widehat{d \sigma}_{\sqrt{\omega}} * \mathcal{R} \mathbf{g}\right) \frac{d \omega}{2 \omega} .
$$

Proof of Theorem 1.6. This theorem requires the estimates for the whole resolvent. Causality suggests the use of the outgoing resolvent to obtain the solution of the forward wave problem (1.33). In a similar fashion the solution of the backward problem would be given by the incoming resolvent. In fact, it is clear, using Fourier transform and assuming $\mathbf{F}$ to be in an appropriate space, that

$$
\mathbf{u}(x, t)=\lim _{\epsilon \rightarrow 0-} \int_{\mathbb{R}} e^{i t \tau}\left(\Delta^{*}+(\tau+i \epsilon)^{2} \mathbf{I}\right)^{-1}((\mathbf{G}(\cdot, \cdot)) \hat{)}(\tau))(x) d \tau,
$$

where $\mathbf{G}(x, t)=\mathbf{F}(x, t) \chi_{(0, \infty)}(t)$, is solution of (1.33). The only thing that remains to be proved is that this solution satisfies the initial conditions given in (1.33).

Using the functional calculus and complex integration, since $\mathbf{G}(x, t)=\mathbf{0}$ for $t<0$, we have that

$$
\begin{aligned}
\mathbf{u}(x, 0) & =\lim _{\epsilon \rightarrow 0-} \int_{\mathbb{R}}\left(\Delta^{*}+(\tau+i \epsilon)^{2} \mathrm{I}\right)^{-1}((\mathbf{G}(\cdot, \cdot) \hat{)}(\tau))(x) d \tau \\
& =\lim _{\epsilon \rightarrow 0-} \int_{\mathbb{R}} \int_{\mathbb{R}} e^{-i t \tau} \int_{0}^{\infty} \frac{1}{-\omega+(\tau+i \epsilon)^{2}} d P_{\omega}(\mathbf{G}(\cdot, t))(x) d \tau d t \\
& =\int_{\mathbb{R}} \int_{0}^{\infty} \lim _{\epsilon \rightarrow 0-} \int_{\mathbb{R}} e^{-i t \tau} \frac{1}{-\omega+(\tau+i \epsilon)^{2}} d \tau d P_{\omega}(\mathbf{G}(\cdot, t))(x) d t \\
& =\int_{\mathbb{R}} \int_{0}^{\infty} \frac{\sin (t \sqrt{\omega})}{2 \sqrt{\omega}} \chi_{(-\infty, 0)}(t) d P_{\omega}(\mathbf{G}(\cdot, t))(x) d t=\mathbf{0} .
\end{aligned}
$$

In a similar way it can be proved that $\mathbf{u}_{t}(x, 0)=\mathbf{0}$.

On the other hand,

$$
\begin{aligned}
D_{x}^{1 / 2} \mathbf{u}(x, t) & =\lim _{\epsilon \rightarrow 0-} \int_{\mathbb{R}} e^{i t \tau} D_{x}^{1 / 2}\left(\Delta^{*}+(\tau+i \epsilon)^{2} \mathbf{I}\right)^{-1}((\mathbf{G}(\cdot, \cdot) \hat{)}(\tau))(x) d \tau \\
& =\lim _{\epsilon \rightarrow 0-}\left(\mathbf{H}_{\epsilon}(\cdot, x) \hat{)}(-t),\right.
\end{aligned}
$$

where $\mathbf{H}_{\epsilon}(\tau, x)=D_{x}^{1 / 2}\left(\Delta^{*}+(\tau+i \epsilon)^{2} \mathbf{I}\right)^{-1}((\mathbf{G}(\cdot, \cdot) \hat{)}(\tau))(x)$. 
Using Plancherel's identity, Fubini and (1.26) we can write

$$
\begin{aligned}
& \sup _{x_{0} \in \mathbb{R}^{n}, R>0} \frac{1}{R} \int_{B\left(x_{0}, R\right)} \int_{\mathbb{R}} \mid\left(\left.\mathbf{H}_{\epsilon}(\cdot, x) \hat{)}(-t)\right|^{2} d t d x\right. \\
& =\sup _{x_{0} \in \mathbb{R}^{n}, R>0} \frac{1}{R} \int_{B\left(x_{0}, R\right)} \int_{\mathbb{R}} \mid D_{x}^{1 / 2}\left(\Delta^{*}+(\tau+i \epsilon)^{2} \mathrm{I}\right)^{-1}\left(\left(\left.\mathbf{G}(\cdot, \cdot) \hat{)(\tau))(x)}\right|^{2} d \tau d x\right.\right. \\
& \leq C\|V\|_{\mathcal{L}^{2, r}} \int_{\mathbb{R}} \int_{\mathbb{R}^{n}} \mid\left(\left.(\mathbf{G}(\cdot, \cdot) \hat{)}(\tau))(x)\right|^{2} V^{-1}(x) d x d \tau\right. \\
& =C\|V\|_{\mathcal{L}^{2, r}} \int_{0}^{\infty}\|\mathbf{F}(\cdot, t)\|_{\mathbf{L}^{2}\left(V^{-1}\right)}^{2} d t .
\end{aligned}
$$

The result follows taking the limit as $\epsilon \rightarrow 0-$ and, using (3.26) and (1.26).

\section{Appendix}

In this section we will show that estimate (1.23) does not hold for $\tilde{r} \leq 2$. To do so, we will prove that:

$$
\left\|\mathbf{R}\left(\omega^{2}+i 0\right) \mathbf{f}\right\|_{\mathbf{L}^{2}(V)} \leq c \omega^{-1}\|V\|_{D_{\tilde{r}}}\|\mathbf{f}\|_{\mathbf{L}^{2}\left(V^{-1}\right)},
$$

does not hold for $\tilde{r} \leq 2$, where $\mathbf{R}\left(\omega^{2}+i 0\right) \mathbf{f}$ is the solution of equation (1.8) given by (1.15).

From (1.18), it is enough to prove the result for $\tilde{r}=2$.

We will consider the case where the Navier equation (1.8) is the Helmholtz equation (1.11), that is, the case where $\mu+\lambda=0$ in (1.2), $\mathbf{f}$ is a scalar function $g$ and $\omega=k$.

Taking into account the following identity, which can be obtained from (2.12) and (3.17),

$R\left(k^{2}+i 0\right) g(x)=$ p.v. $\int_{\mathbb{R}^{n}} \frac{1}{-|\xi|^{2}+k^{2}} \widehat{g}(\xi) e^{i x \cdot \xi} d \xi+\frac{i \pi}{2 k} \chi_{\{k>0\}} \widehat{d \sigma_{k}} * g(x), \quad x \in \mathbb{R}^{n}$,

we claim that it is enough to prove that the following estimate does not hold:

$$
\left\|\widehat{d \sigma_{k}} * g\right\|_{L^{2}(V)} \leq c\|V\|_{D_{2}}\|g\|_{L^{2}\left(V^{-1}\right)} .
$$

A scaling argument shows that we may assume $k=1$ without loss of generality.

Notation. Throughout this section, for nonnegative quantities $X$ and $Y$ we will write $X \lesssim Y(X \gtrsim Y)$ to denote the existence of a positive constant $C$, depending only on the dimension $n$, such that $X \leq C Y(X \geq C Y)$. We will write $X \sim Y$ if both $X \lesssim Y$ and $X \gtrsim Y$ hold.

The following lemma proves that (4.1) does not hold. 
Theorem 4.1. Given a natural number $N$ sufficiently large, there exist a nonnegative radial function $V_{N}$ in $D_{2}\left(\mathbb{R}^{n}\right), n \geq 2$, with $\left\|V_{N}\right\|_{D_{2}}=1$, and a function $g_{N}$ in $L^{2}\left(V_{N}^{-1}\right)$ such that

$$
\left\|\widehat{d \sigma} * g_{N}\right\|_{L^{2}\left(V_{N}\right)}^{2} \gtrsim \log N\left\|g_{N}\right\|_{L^{2}\left(V_{N}^{-1}\right)}^{2},
$$

where do denotes the induced Lebesgue measure on the unit sphere.

In order to prove Theorem 4.1 we need the following technical results that can be found in [4, 10] and [3] (see [4, Lemma 5] or estimate [10, (2.4.2)] and, [3, Section 2]).

Lemma 4.2. For $k=0,1,2, \ldots$, let $\left\{Y_{m}^{k} / m=1,2, \ldots, a_{k}\right\}$ be an orthonormal basis of spherical harmonics of degree $k$. Given a nonnegative radial function $V$ and a function $g$ in $L^{2}\left(\mathbb{R}^{n}\right), n \geq 2$, we have that

$$
\begin{aligned}
& \|\widehat{d \sigma} * g\|_{L^{2}\left(V_{N}\right)}^{2} \\
& =\sum_{k=0}^{\infty} \sum_{m=1}^{a_{k}}\left|\int_{0}^{\infty} t^{n / 2} J_{v(k)}(t) g_{k m}(t) d t\right|^{2} \int_{0}^{\infty}\left|J_{v(k)}(r)\right|^{2} r V(r) d r,
\end{aligned}
$$

where $v(k)=k+(n-2) / 2, J_{v}$ denotes the Bessel function of order $v$, and $g_{k m}$ are the coefficients of $g$ in its spherical harmonic expansion, i.e.

$$
g(x)=\sum_{k=0}^{\infty} \sum_{m=1}^{a_{k}} g_{k m}(|x|) Y_{m}^{k}\left(\frac{x}{|x|}\right), \quad x \in \mathbb{R}^{n} \text {. }
$$

Lemma 4.3. Given $v \geq 1 / 2$, let $K$ be the unique natural number such that $v^{2 / 3} \leq$ $2^{K} \leq 2 v^{2 / 3}$. Then, for $1 \leq p<\infty$, we have that

$$
\int_{v+v^{1 / 3}}^{2 v}\left|J_{v}(r)\right|^{p} d r \sim v^{(1-p) / 3} \sum_{j=0}^{K} 2^{j(1-p / 4)} .
$$

Lemma 4.4. Given $v \geq 1 / 2$, there exists a positive constant $A$ independent of $v$ such that if $r \in\left[v+v^{1 / 3}, 2 v\right]$, then

$$
J_{v}(r)=\frac{\cos \theta_{v}(r)}{\sqrt{2 \pi}\left(r^{2}-v^{2}\right)^{1 / 4}}+\gamma_{\nu}(r),
$$

where

$$
\theta_{v}(r)=\left(r^{2}-v^{2}\right)^{1 / 2}-v \arccos \left(\frac{v}{r}-\frac{\pi}{4}\right)
$$

and

$$
\left|\gamma_{v}(r)\right| \leq A\left(\frac{v^{2}}{\left(r^{2}-v^{2}\right)^{7 / 4}}+\frac{1}{r}\right)
$$


Proof of Theorem 4.1. For $N$ is sufficiently large, we can write $v=v(N)=$ $v(\ell)=\ell+(n-2) / 2$ with $\ell \in \mathbb{N}$ such that $2^{N} \leq v \leq 2^{N+1}$.

For $|x| \in\left[2^{N}, 2^{N+2}\right]$, let $V_{N}(x)=C_{N} h_{N}(|x|)$ where

$$
h_{N}(r)=|r-v|^{-1 / 2}\left[\log \left(\frac{4 v}{|r-v|}\right)\right]^{-1} \chi_{\left[2^{N}, 2^{N+2}\right]}(r)
$$

and $C_{N}=1 /\left(2\left\|h_{N}\right\|_{D_{2}}\right)$; and for $|x| \notin\left[2^{N}, 2^{N+2}\right]$, we define $V_{N}(x)$ as a nonnegative radial function such that $V_{N} \in D_{2}\left(\mathbb{R}^{n}\right)$ and $\left\|V_{N}\right\|_{D_{2}}=1$.

Observe that since $\left\|h_{N}\right\|_{D_{2}} \sim 2^{N / 2} \sim v^{1 / 2}$, then

$$
C_{N} \sim v^{-1 / 2}
$$

For $x \in \mathbb{R}^{n}$, we also define,

$$
g_{N}(x)=\chi_{\left[v+v^{1 / 3}, 2 v\right]}(|x|) \operatorname{sign}\left(J_{v}(|x|)\right) Y_{1}^{\ell}\left(\frac{x}{|x|}\right) .
$$

It is easy to see that

$$
\left\|g_{N}\right\|_{L^{2}\left(V_{N}^{-1}\right)}^{2} \lesssim v^{n+1}
$$

and therefore, it is enough to prove that

$$
\left\|\widehat{d \sigma} * g_{N}\right\|_{L^{2}\left(V_{N}\right)}^{2} \gtrsim \log N v^{n+1} .
$$

Using (4.2) and (4.3) with $p=1$, for this particular function $g_{N}$, we can write

$$
\begin{aligned}
\left\|\widehat{d \sigma} * g_{N}\right\|_{L^{2}\left(V_{N}\right)}^{2} & =\left(\int_{\nu+v^{1 / 3}}^{2 v} t^{n / 2}\left|J_{v}(t)\right| d t\right)^{2} \int_{0}^{\infty}\left|J_{v}(r)\right|^{2} r V_{N}(r) d r \\
& \sim v^{n}\left(\sum_{j=0}^{K} 2^{3 j / 4}\right)^{2} \int_{0}^{\infty}\left|J_{v}(r)\right|^{2} r V_{N}(r) d r
\end{aligned}
$$

where $K$ is the unique natural number such that $v^{2 / 3} \leq 2^{K} \leq 2 v^{2 / 3}$. Therefore,

$$
\begin{aligned}
\left\|\widehat{d \sigma} * g_{N}\right\|_{L^{2}\left(V_{N}\right)}^{2} & \sim v^{n+1} \int_{0}^{\infty}\left|J_{v}(r)\right|^{2} r V_{N}(r) d r \\
& \gtrsim v^{n+2} \sum_{j=0}^{K-2} \int_{v+2^{j} v^{1 / 3}}^{v+2^{j+1} v^{1 / 3}}\left|J_{v}(r)\right|^{2} V_{N}(r) d r .
\end{aligned}
$$

Using (4.4) and (4.7), for this particular function $V_{N}$, we have that

$$
\left\|\widehat{d \sigma} * g_{N}\right\|_{L^{2}\left(V_{N}\right)}^{2} \gtrsim v^{n+2 / 3} \sum_{j=0}^{K-2} 2^{-j}\left[\log \left(v^{2 / 3} 2^{-j}\right)\right]^{-1} I_{j},
$$


where

$$
I_{j}=\int_{\nu+2^{j} v^{1 / 3}}^{v+2^{j+1} v^{1 / 3}}\left|\cos \theta(r)+\sqrt{2 \pi}\left(r^{2}-v^{2}\right)^{1 / 4} h_{v}(r)\right|^{2} d r, \quad j=0, \ldots, K-2 .
$$

For each $j$, we define

$$
B_{j}=\left\{r \in\left[v+2^{j} v^{1 / 3}, v+2^{j+1} v^{1 / 3}\right]:\left|\cos \theta_{v}(r)\right| \geq 1 / 2\right\}
$$

where $\theta_{v}(r)$ is given by (4.5).

For any natural number $m$ such that $m \leq K-2$ (this is possible for $N$ large enough), using (4.6), one can prove that if $r \in\left[v+2^{m} v^{1 / 3}, v+2^{K-1} v^{1 / 3}\right]$, then

$$
\left|\sqrt{2 \pi}\left(r^{2}-v^{2}\right)^{1 / 4} h_{v}(r)\right| \leq 4 \sqrt{\pi} A 2^{-3 m / 4} .
$$

Therefore, if we choose the natural number $m$ such that $4 \sqrt{\pi} A 2^{-3 m / 4} \leq 1 / 4$ (again, this is possible for $N$ large enough), from (4.9), (4.10) and (4.11), we have that

$$
I_{j} \gtrsim \int_{B_{j}} d r \sim 2^{j} v^{1 / 3}, \quad j=m, \ldots, K-2 .
$$

Using this estimate in (4.8), and the fact that $v^{2 / 3} \sim 2^{K} \sim 2^{2 N / 3}$, we get that

$$
\left\|\widehat{d \sigma} * g_{N}\right\|_{L^{2}\left(V_{N}\right)}^{2} \gtrsim v^{n+1} \sum_{j=m}^{K-2}\left(\log 2^{K-j}\right)^{-1} \sim v^{n+1} \log K \sim v^{n+1} \log N
$$

whenever $K \geq m / 2$ (as before, this is possible for $N$ large enough).

Remark 4.5. Notice that the weights $V_{N}$ defined in the proof of Theorem 4.1 do not belong to $D_{p}$ if $p>2$.

Remark 4.6. [4, Theorem 3] gives a characterization of the weights that belong to $\mathcal{T}$ in terms of a restriction theorem for spheres. More precisely, Theorem 3 states that for $n \geq 2, V \in \mathcal{T}$ if and only if

$$
\left\|\widehat{d \sigma_{k}} * g\right\|_{L^{2}(V)} \lesssim\|\| V\|\|\|\|_{L^{2}\left(V^{-1}\right)}
$$

As a consequence of Theorem 4.1 and (4.12), we have that if $p \leq 2, D_{p}$ is not continuously included in $\mathcal{T}$. 


\section{References}

[1] S. Agmon, Spectral properties of Schrödinger operators and scattering theory, Ann. Scuola Norm. Sup. Pisa (4) 2 (1975), 151-218.

[2] S. AGMON and L. HÖRMANDER, Asymptotic properties of solutions of differential equations with simple characteristics, J. Anal. Math. 30 (1976), 1-38.

[3] J. A. BARCELÓ and A. CÓRDOBA, Band-limited functions: $L^{p}$-convergence, Trans. Amer. Math. Soc. (2) 313 (1989), 655-669.

[4] J. A. BARCELÓ, A. RUIZ and L. VeGA, Weighted estimates for the Helmholtz equation and some applications, J. Funct. Anal. 150 (1997), 356-382.

[5] A. P. CAlderón and A. ZYgmund, On singular integrals, Amer. J. Math. 78 (1956), 289-309.

[6] S. Campanato, Proprietà di una famiglia di spazi funzionali, Ann. Scuola Norm. Sup. Pisa 18 (1964), 137-160.

[7] F. ChiAREnZA and M. FrasCA, A remark on a paper by C. Fefferman, Proc. Amer. Math. Soc. 108 (1990), 407-409.

[8] F. Chiarenza and A. Ruiz, Uniform $L^{2}$-weighted Sobolev inequalities, Proc. Amer. Math. Soc. 112 (1991), 53-64.

[9] R. R. COIFMAN and C. FEFFERMAN, Weighted norm inequalities for maximal functions and singular integrals, Studia Math. 51 (1974), 241-250.

[10] D. Colton and R. KRess, "Inverse Acoustic and Electromanetic Scattering Theory", Springer-Verlag, New York, 1992.

[11] J. DuOAndikoetxea Zuazo, "Fourier Analysis", AMS, Graduate Studies in Mathematics, V 29, 2001.

[12] J. García-Cuerva and J. L. Rubio De Francia, "Weighted Norms Inequalities and Related Topics", North-Holland, Amsterdam, 1985.

[13] C. HERZ, Lipschitz spaces and Bernstein's theorem on absolutely convergent Fourier transforms, J. Math. Mech. 18 (1968), 283-324.

[14] G. HU, S. LU and D. YANG, Boundedness of rough singular integral operators on homogeneous Herz spaces, J. Austr. Math. Soc. 66 (1999), 201-223.

[15] T. KATO, Wave operators and similarity for non-selfadjoint operators, Math. Ann. 162 (1966), 258-279.

[16] T. Kato and K. YAJima, Some examples of smooth operators and the associated smoothing effect, Rev. Math. Phy. (4) 1 (1989), 481-496.

[17] C. Kenig, G. Ponce and L. VegA, Small solutions to nonlinear Schrödinger equations, Ann. Inst. H. Poincaré Anal. Non Linéaire 10 (1993), 255-288.

[18] C. Kenig, A. RUIZ and C. SogGe, Uniform Sobolev inequalities and unique continuation for second order constant coefficient differential operators, Duke Math. J. 55 (1987), 329_ 347.

[19] V. D. Kupradze, "Three-Dimensional Problems of Elasticity and Thermoelasticity", North-Holland, Amsterdam, 1979.

[20] R. LEIS, "Initial Boundary Value Problems in Mathematical Physics", John Wiley \& Sons, New York, 1986.

[21] X. LI and D. YANG, Boundedness of some sublinear operators on Herz spaces, Illinois J. Math. 40 (1996), 484-501.

[22] B. Perthame and L. Vega, Morrey-Campanato estimates for Helmholtz equations, J. Funct. Anal. 164 (1999), 340-355.

[23] A. RUIZ, Recovery of the singluarities of a potential from fixed angle scattering data, Comm. Partial Differential Equations 26 (2001), 1721-1738.

[24] A. RuIZ, Harmonic analysis and inverse problems, http://web.uam.es/gruposinv/inversos/publicaciones/Inverseproblems.pdf

[25] A. RuIZ and A. VARgas, Partial recovery of a potential from backscattering data, Comm. Partial Differential Equations 30 (2005), 67-96. 
[26] A. RUIZ and L. VEGA, Unique continuation for Schrödinger equations with potential in Morrey classes, Publ. Math. 35 (1991), 291-298.

[27] A. RUIZ and L. VeGA, On local regularity of Schrödinger equations, Duke Math. J. 76 (1994), 913-940.

[28] A. RUIZ and L. VEGA, Local regularity of solutions to wave equations with time-dependent potentials, Int. Math. Res. Not. INRN 1 (1993), 13-27.

[29] G. Stampacchia, $\mathcal{L}^{p, \lambda}$-Spaces and interpolation, Comm. Pure Appl. Math. 17 (1964), 293-306.

[30] L.Vega and N. Visciglia, On the local smoothing for the Schrödinger equation, Proc. Amer. Math. Soc. 135 (2007), 119-128.

ETSI de Caminos

Universidad Politécnica de Madrid

28040 Madrid, Spain

juanantonio.barcelo@upm.es

Instituto de Matemáticas

Universidad Nacional Autónoma de México

Ciudad Universitaria

México D.F., 04510, México

folchgab@matem.unam.mx

Instituto de Matemáticas Unidad Cuernavaca Universidad Nacional Autónoma de México A.P. 273-3 ADMON 3

Cuernavaca, Mor. - 62251, México

salvador@matcuer.unam.mx

Departamento de Matemáticas

Universidad Autónoma de Madrid

28049, Madrid, Spain

alberto.ruiz@uam.es

Departamento de Matemática Aplicada

Universidad de Valladolid

Plaza Santa Eulalia 9 y 11

40005 Segovia, Spain

maricruz@dali.eis.uva.es 